\title{
A Pair of Three-Step Hybrid Block Methods for the Solutions of Linear and Nonlinear First-Order Systems
}

\author{
Joshua Sunday, Chibuisi Chigozie, Ezekiel O. Omole and John B. Gwong
}

\begin{abstract}
In this research paper, a pair of three-step hybrid block methods is derived for the solutions of linear and nonlinear first-order systems. The derivation is carried out with the aid of collocation and interpolation technique and the adoption of power series as basis function. The first and second three-step hybrid block methods are derived by incorporating a single and double off-grid point(s) respectively within the three-step integration interval. The methods derived were then applied on some linear and nonlinear first-order systems to test their accuracy and efficiency. The results obtained show that the three-step hybrid block method with two offgrid points performed better than the three-step hybrid block method with one off-grid point. It was also clear from the results obtained that the two methods derived performed better than the existing methods with which we compared our results. We further analyzed the basic properties of the methods derived. These properties include zero-stability, consistence, convergence and region of absolute stability.
\end{abstract}

Keywords — First-order, hybrid, linear, nonlinear, off-grid, three-step.

\section{INTRODUCTION}

In this paper, we shall derive a pair of three-step methods for the solution of linear and nonlinear first order systems of the form,

$$
y^{\prime}(x)=f(x, y), y\left(x_{0}\right)=y_{0}
$$

where $f: \mathfrak{R} \times \mathfrak{R}^{2 q} \rightarrow \mathfrak{R}^{q} ; y, y_{0} \in \mathfrak{R}^{q}$, and $q$ is the dimension of the system. The function $f$ is assumed to satisfy the Lipschitz condition stated in the Theorem below.

Theorem 1 [1]

Let $f(x, y)$ be a function, defined and continuous for all points $(x, y)$ in the region $D$ defined by $a \leq x \leq b,-\infty<y<\infty), a$ and $b$ finite, and let there exist a constant $L$ such that, for every $x, y, y^{*}$ such that $(x, y)$ and $f\left(x, y^{*}\right)$ are both in $D$,

$$
\left|f(x, y)-f\left(x, y^{*}\right)\right| \leq L\left|y-y^{*}\right|
$$

Then, if $\eta$ is any given number, there exists a unique solution $y(x)$ of the initial value problem (1), where $y(x)$ is continuous and differentiable for all $(x, y)$ in $D$. The requirement (2) is known as a Lipschitz condition and the constant $L$ as a Lipschitz constant.

A lot of algorithms and methods have been proposed by scholars for the solution of first-order stiff systems of the form (1), these authors among others include [2]-[9].

Published on January 24, 2022

J. Sunday, Department of Mathematics, University of Jos, Nigeria.

(corresponding e-mails: sundayjo@unijos.edu.ng, joshuasunday2000@yahoo.com)

C. Chigozie, Department of Insurance, University of Jos, Nigeria.

(e-mail: chigoziec@unijos.edu.ng)

E.O. Omole, Department of Mathematics \& Statistics, Joseph Ayo Babalola University, Osogbo, Nigeria.

(e-mail: omolez247@gmail.com)

J.B. Gwong, Department of Mathematics, University of Jos, Nigeria.

(e-mail: botjohn15@gmail.com) 
However, it is important to state that these methods have some setbacks ranging from small convergence/implementation region to inefficiency in terms of accuracy. In view of these setbacks, we are motivated to formulate a pair of three-step hybrid block methods that will address some of these setbacks. The proposed methods have the advantage of generating simultaneous numerical approximations at different grid points within the interval of integration. Another advantage of the methods is that they are less expensive in terms of the number of function evaluations compared to the conventional linear multistep and the Runge-Kutta methods. They also preserve the traditional advantage of one-step methods of being self-starting and permitting easy change of step-size during integration, [10].

\section{Derivation of the Three-Step Hybrid Block Methods}

In this section, a pair of three-step hybrid block methods of the form,

$$
A^{(0)} Y_{m}=E y_{n}+h d f\left(y_{n}\right)+h b f\left(Y_{m}\right)
$$

where $A^{(0)}, E, d$ and $b$ are $(r-1) \times(r-1)$ matrices shall be derived for the solution of linear and nonlinear first order systems of the form (1). To achieve this, we approximate the exact solution $y(x)$ to (1) by assuming an approximate solution $Y(x)$ in the form,

$$
Y(x)=\sum_{i=0}^{r+s-1} p_{i} \omega_{i}(x)
$$

where $r$ and $s$ are the numbers of collocation and interpolation points respectively, $x \in\left[x_{0}, x_{n}\right], p_{i}$ are undetermined coefficients that must be obtained and $\omega_{i}(x)$ are basis polynomial function of degree $r+s-1$.

\section{A. Three-Step Hybrid Block Method with One Off-Grid Point}

To derive the three-step hybrid block method with one off-grid point, we carryout interpolation at $x_{n+s}, s=\frac{1}{2}$ and collocation at $x_{n+r}, r=0, \frac{1}{2}, 1,2,3$ as follows,

$$
\begin{aligned}
& \sum_{j=0}^{5} p_{j} x_{n+s}^{j}=y_{n+s}, s=\frac{1}{2} \\
& \sum_{j=0}^{5} j p_{j} x_{n+r}^{j-1}=f_{n+r}, r=0, \frac{1}{2}, 1,2,3
\end{aligned}
$$

Equations (5) and (6) gives a system of nonlinear equation of the form

$$
X A=U
$$

Where,

$$
A=\left[\begin{array}{llllll}
a_{0} & a_{1} & a_{2} & a_{3} & a_{4} & a_{5}
\end{array}\right]^{T}, \quad U=\left[\begin{array}{llllll}
y_{n} & f_{n} & f_{n+\frac{1}{2}} & f_{n+1} & f_{n+2} & f_{n+3}
\end{array}\right]^{T}
$$




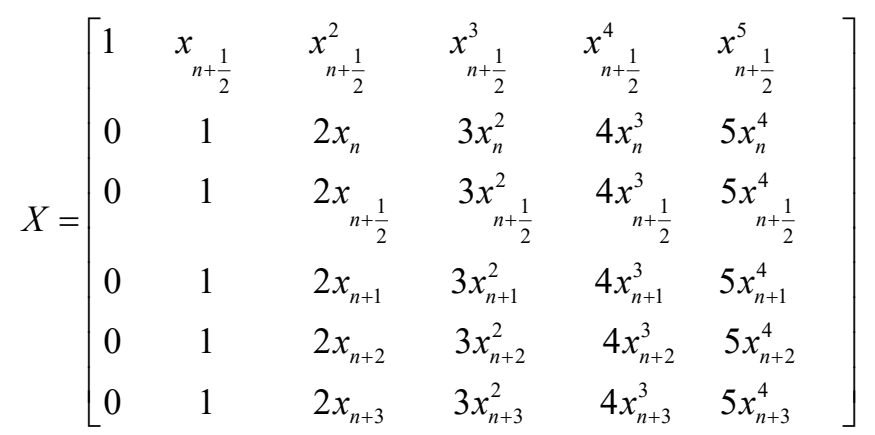

Solving the system of nonlinear equation by Gauss elimination method for the $p_{j}{ }^{\prime} s, j=0(1) 5$ and substituting back into (4) gives a continuous three-step hybrid block method with one off-grid point of the form,

$$
Y(x)=\alpha_{\frac{1}{2}}(x) y_{n+\frac{1}{2}}+h\left(\sum_{j=0}^{3} \beta_{k}(x) f_{n+k}+\beta_{\frac{1}{2}}(x) f_{n+\frac{1}{2}}\right)
$$

(8)

where

$$
\left.\begin{array}{l}
\alpha_{\frac{1}{2}}(x)=1 \\
\beta_{0}(x)=\frac{1}{5760}\left(384 t^{5}-3120 t^{4}+8960 t^{3}-11040 t^{2}+5760 t-1057\right) \\
\beta_{\frac{1}{2}}(x)=-\frac{1}{225}\left(48 t^{5}-360 t^{4}+880 t^{3}-720 t^{2}+91\right) \\
\beta_{1}(x)=-\frac{1}{1920}\left(384 t^{5}-2640 t^{4}+5440 t^{3}-2880 t^{2}+193\right) \\
\beta_{2}(x)=-\frac{1}{5760}\left(384 t^{5}-2160 t^{4}+3200 t^{3}-1440 t^{2}+83\right) \\
\beta_{3}(x)=\frac{1}{28800}\left(384 t^{5}-1680 t^{4}+2240 t^{3}-960 t^{2}+53\right)
\end{array}\right\}
$$

and $t$ is given by

$$
t=\frac{x-x_{n}}{h}
$$

Evaluating (8) at $t=\frac{1}{2}, 1,2,3$ gives the new discrete three-step hybrid block method with one off-grid point of the form (3) as 


$$
\begin{aligned}
{\left[\begin{array}{llll}
1 & 0 & 0 & 0 \\
0 & 1 & 0 & 0 \\
0 & 0 & 1 & 0 \\
0 & 0 & 0 & 1
\end{array}\right]\left[\begin{array}{l}
y_{n+\frac{1}{2}} \\
y_{n+1} \\
y_{n+2} \\
y_{n+3}
\end{array}\right] } & {\left[\begin{array}{llll}
0 & 0 & 0 & 1 \\
0 & 0 & 0 & 1 \\
0 & 0 & 0 & 1 \\
0 & 0 & 0 & 1
\end{array}\right]\left[\begin{array}{l}
y_{n-\frac{1}{2}} \\
y_{n-1} \\
y_{n-2} \\
y_{n}
\end{array}\right]+h\left[\begin{array}{cccc}
0 & 0 & 0 & \frac{1057}{5760} \\
0 & 0 & 0 & \frac{59}{360} \\
0 & 0 & 0 & \frac{11}{45} \\
0 & 0 & 0 & \frac{3}{40}
\end{array}\right]\left[\begin{array}{l}
f_{n-\frac{1}{2}} \\
f_{n-1} \\
f_{n-2} \\
f_{n}
\end{array}\right] } \\
+ & h\left[\begin{array}{llll}
\frac{91}{225} & -\frac{193}{1920} & \frac{83}{5760} & -\frac{53}{28800} \\
\frac{152}{225} & \frac{19}{120} & \frac{1}{360} & -\frac{1}{1800} \\
\frac{64}{225} & \frac{16}{15} & \frac{19}{45} & -\frac{4}{225} \\
\frac{24}{25} & \frac{9}{40} & \frac{57}{40} & \frac{63}{200}
\end{array}\right]\left[\begin{array}{l}
f_{n+\frac{1}{2}} \\
f_{n+1} \\
f_{n+2} \\
f_{n+3}
\end{array}\right]
\end{aligned}
$$

\section{B. Three-Step Hybrid Block Method with Two Off-Grid Points}

For three-step hybrid block method with two off-grid points, we interpolate at $x_{n+s}, s=\frac{3}{2}$ and collocate at $x_{n+r}, r=0, \frac{1}{2}, 1, \frac{3}{2}, 2,3$. This gives,

$$
\begin{aligned}
& \sum_{j=0}^{6} p_{j} x_{n+s}^{j}=y_{n+s}, s=\frac{3}{2} \\
& \sum_{j=0}^{6} j p_{j} x_{n+r}^{j-1}=f_{n+r}, r=0, \frac{1}{2}, 1, \frac{3}{2}, 2,3
\end{aligned}
$$

Equations (12) and (13) will together give a system nonlinear equation of the form (7), where

$$
\begin{aligned}
& A=\left[\begin{array}{lllllll}
a_{0} & a_{1} & a_{2} & a_{3} & a_{4} & a_{5} & a_{6}
\end{array}\right]^{T}, \quad U=\left[\begin{array}{lllllll}
y_{n} & f_{n} & f_{n+\frac{1}{2}} & f_{n+1} & f_{n+\frac{3}{2}} & f_{n+2} & f_{n+3}
\end{array}\right]^{T} \\
& X=\left[\begin{array}{ccccccc}
1 & x_{n+\frac{3}{2}} & x_{n+\frac{3}{2}}^{2} & x_{n+\frac{3}{2}}^{3} & x_{n+\frac{3}{2}}^{4} & x_{n+\frac{3}{2}}^{5} & x_{n+\frac{3}{2}}^{6} \\
& 1 & 2 x_{n} & 3 x_{n}^{2} & 4 x_{n}^{3} & 5 x_{n}^{4} & 6 x_{n}^{5} \\
0 & 1 & 2 x_{n+\frac{1}{2}} & 3 x_{n+\frac{1}{2}}^{2} & 4 x_{n+\frac{1}{2}}^{3} & 5 x_{n+\frac{1}{2}}^{4} & 6 x_{n+\frac{1}{2}}^{5} \\
0 & 1 & 2 x_{n+1} & 3 x_{n+1}^{2} & 4 x_{n+1}^{3} & 5 x_{n+1}^{4} & 6 x_{n+1}^{5} \\
0 & 1 & 2 x_{n+\frac{3}{2}} & 3 x^{2} & 4 x^{3} & 5 x^{4} \frac{3}{2} & 6 x^{5} \\
0 & 1 & 2 x_{n+2} & 3 x_{n+2}^{2} & 4 x_{n+2}^{3} & 5 x_{n+2}^{4} & 6 x_{n+2}^{5} \\
0 & 1 & 2 x_{n+3} & 3 x_{n+3}^{2} & 4 x_{n+3}^{3} & 5 x_{n+3}^{4} & 6 x_{n+3}^{5}
\end{array}\right]
\end{aligned}
$$

Similarly, solving the system of nonlinear equation by Gauss elimination method for the $p_{j}{ }^{\prime} s, j=0(1) 6$ and substituting back into (4) gives a continuous three-step hybrid method with two offgrid points of the form,

$$
Y(x)=\alpha_{\frac{3}{2}}(x) y_{n+\frac{3}{2}}+h\left(\sum_{j=0}^{3} \beta_{k}(x) f_{n+k}+\beta_{\frac{1}{2}}(x) f_{n+\frac{1}{2}}+\beta_{\frac{3}{2}}(x) f_{n+\frac{3}{2}}\right)
$$

where 


$$
\left.\begin{array}{l}
\alpha_{\frac{3}{2}}(x)=1 \\
\beta_{0}(x)=-\frac{1}{17280}\left(640 t^{6}-6144 t^{5}+22800 t^{4}-41600 t^{3}+38880 t^{2}-17280 t+2781\right) \\
\beta_{\frac{1}{2}}(x)=\frac{1}{360}\left(64 t^{6}-576 t^{5}+1920 t^{4}-2880 t^{3}+1728 t^{2}-243\right) \\
\beta_{1}(x)=-\frac{1}{1920}\left(640 t^{6}-5376 t^{5}+16080 t^{4}-20160 t^{3}+8640 t^{2}+729\right) \\
\beta_{\frac{3}{2}}(x)=\frac{1}{1080}\left(320 t^{6}-2496 t^{5}+6720 t^{4}-7360 t^{3}+2880 t^{2}-351\right) \\
\beta_{2}(x)=-\frac{1}{5760}\left(640 t^{6}-4608 t^{5}+11280 t^{4}-11520 t^{3}+4320 t^{2}-243\right) \\
\beta_{3}(x)=\frac{1}{17280}\left(128 t^{6}-768 t^{5}+1680 t^{4}-1600 t^{3}+576 t^{2}-27\right)
\end{array}\right\}
$$

and $t$ as defined in (10). Evaluating (14) at $t=\frac{1}{2}, 1, \frac{3}{2}, 2,3$ gives the new discrete three-step hybrid block method with two off-grid points of the form (3) as,

$$
\begin{aligned}
& {\left[\begin{array}{lllll}
1 & 0 & 0 & 0 & 0 \\
0 & 1 & 0 & 0 & 0 \\
0 & 0 & 1 & 0 & 0 \\
0 & 0 & 0 & 1 & 0 \\
0 & 0 & 0 & 0 & 1
\end{array}\right]\left[\begin{array}{l}
y_{n+\frac{1}{2}} \\
y_{n+1} \\
y_{n+\frac{3}{2}} \\
y_{n+2} \\
y_{n+3}
\end{array}\right] } {\left[\begin{array}{lllll}
0 & 0 & 0 & 0 & 1 \\
0 & 0 & 0 & 0 & 1 \\
0 & 0 & 0 & 0 & 1 \\
0 & 0 & 0 & 0 & 1 \\
0 & 0 & 0 & 0 & 1
\end{array}\right]\left[\begin{array}{l}
y_{n-\frac{1}{2}} \\
y_{n-1} \\
y_{n-\frac{3}{2}} \\
y_{n-2} \\
y_{n}
\end{array}\right]+h\left[\begin{array}{ccccc}
0 & 0 & 0 & 0 & \frac{959}{5760} \\
0 & 0 & 0 & 0 & \frac{169}{1080} \\
0 & 0 & 0 & 0 & \frac{103}{640} \\
0 & 0 & 0 & 0 & \frac{7}{45} \\
0 & 0 & 0 & 0 & \frac{11}{40}
\end{array}\right]\left[\begin{array}{l}
f_{n-\frac{1}{2}} \\
f_{n-1} \\
f_{n-\frac{3}{2}} \\
f_{n-2} \\
f_{n}
\end{array}\right] } \\
&+h\left[\begin{array}{cccccc}
\frac{35}{72} & -\frac{487}{1920} & \frac{49}{360} & -\frac{211}{5760} & \frac{1}{640} \\
\frac{32}{45} & \frac{11}{120} & \frac{8}{135} & -\frac{7}{360} & \frac{1}{1080} \\
\frac{27}{40} & \frac{243}{640} & \frac{13}{40} & -\frac{27}{640} & \frac{1}{640} \\
\frac{32}{45} & \frac{4}{15} & \frac{32}{45} & \frac{7}{45} & 0 \\
0 & \frac{81}{40} & -\frac{8}{5} & \frac{81}{40} & \frac{11}{40}
\end{array}\right]\left[\begin{array}{l}
f_{n+\frac{1}{2}} \\
f_{n+1} \\
f_{n+\frac{3}{2}} \\
f_{n+2} \\
f_{n+3}
\end{array}\right]
\end{aligned}
$$

\section{ANAlysis OF THE THREe-STEP Hybrid Block Methods}

In this section, some basic properties of the pair of three-step hybrid block methods shall be analyzed. These properties among others include the order, consistency, zero-stability, convergence and stability region.

\section{A. Order and Error Constants of the Three-Step Hybrid Block Methods}

The linear operator $L\{y(x) ; h\}$ of the three-step method of the form (4) is defined as,

$$
L\{y(x) ; h\}=A^{(0)} Y_{m}-E y_{n}-h d f\left(y_{n}\right)-h b f\left(Y_{m}\right)
$$

Taking the Taylor series expansion of (17) and comparing the coefficients of $h$ gives, 


$$
\begin{aligned}
& L\{y(x) ; h\}=c_{0} y(x)+c_{1} h y^{\prime}(x)+c_{2} h^{2} y^{\prime \prime}(x) \\
& +\ldots+c_{p} h^{p} y^{p}(x)+c_{p+1} h^{p+1} y^{p+1}(x)+\ldots
\end{aligned}
$$

\section{Definition $1[11]$}

A method is said to be of order $p$ if $p$ is the largest positive integer for which $\bar{c}_{0}=\bar{c}_{1}=\bar{c}_{2}=\ldots=\bar{c}_{p}=0, \bar{c}_{P+1} \neq 0$. The term $\bar{c}_{p+1}$ is called the error constant of the method. Suffice to say that the order of a method quantifies the rate of convergence of a numerical approximation of a differential equation to that of the exact solution. On the other hand, the error constant is the accumulated error when the order of a method has been computed.

Therefore, the Taylor series expansion of the three-step hybrid block method with one off-grid point is,

$$
\left[\begin{array}{l}
\sum_{j=0}^{\infty} \frac{\left(\frac{1}{2}\right)^{j}}{j !} y_{n}^{j}-y_{n}-\frac{1057}{5760} h y_{n}^{\prime}-\sum_{j=0}^{\infty} \frac{h^{j+1}}{j !} y_{n}^{j+1}\left\{\frac{91}{225}\left(\frac{1}{2}\right)^{j}-\frac{193}{1920}(1)^{j}+\frac{83}{5760}(2)^{j}-\frac{53}{28800}\right. \\
\sum_{j=0}^{\infty} \frac{(1)^{j}}{j !} y_{n}^{j}-y_{n}-\frac{59}{360} h y_{n}^{\prime}-\sum_{j=0}^{\infty} \frac{h^{j+1}}{j !} y_{n}^{j+1}\left\{\frac{152}{225}\left(\frac{1}{2}\right)^{j}+\frac{19}{120}(1)^{j}+\frac{1}{360}(2)^{j}-\frac{1}{1800}(3)^{j}\right\} \\
\sum_{j=0}^{\infty} \frac{(2)^{j}}{j !} y_{n}^{j}-y_{n}-\frac{11}{45} h y_{n}^{\prime}-\sum_{j=0}^{\infty} \frac{h^{j+1}}{j !} y_{n}^{j+1}\left\{\frac{64}{225}\left(\frac{1}{2}\right)^{j}+\frac{16}{15}(1)^{j}+\frac{19}{45}(2)^{j}-\frac{4}{225}(3)^{j}\right\} \\
\sum_{j=0}^{\infty} \frac{(3)^{j}}{j !} y_{n}^{j}-y_{n}-\frac{3}{40} h y_{n}^{\prime}-\sum_{j=0}^{\infty} \frac{h^{j+1}}{j !} y_{n}^{j+1}\left\{\frac{24}{25}\left(\frac{1}{2}\right)^{j}+\frac{9}{40}(1)^{j}+\frac{57}{40}(2)^{j}+\frac{63}{200}(3)^{j}\right\}
\end{array}\right.
$$

Thus, $\bar{c}_{0}=\bar{c}_{1}=\bar{c}_{2}=\bar{c}_{3}=\bar{c}_{4}=\bar{c}_{5}=\overline{0}$; implying that the order of the three-step method with one offgrid point is $p=\left[\begin{array}{llll}5 & 5 & 5 & 5\end{array}\right]^{T}$. That is, the method is of uniform order 5 .

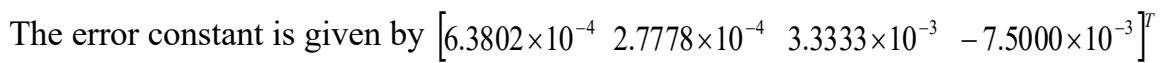

Similarly, the Taylor series expansion of the three-step hybrid block method with two off-grid points is given by,

$$
\left[\begin{array}{l}
\sum_{j=0}^{\infty} \frac{\left(\frac{1}{2}\right)^{j}}{j !} y_{n}^{j}-y_{n}-\frac{959}{5760} h y_{n}^{\prime}-\sum_{j=0}^{\infty} \frac{h^{j+1}}{j !} y_{n}^{j+1}\left\{\frac{35}{72}\left(\frac{1}{2}\right)^{j}-\frac{487}{1920}(1)^{j}+\frac{49}{360}\left(\frac{3}{2}\right)^{j}-\frac{211}{5760}(2)^{j}+\frac{1}{640}(3)^{j}\right\} \\
\sum_{j=0}^{\infty} \frac{(1)^{j}}{j !} y_{n}^{j}-y_{n}-\frac{169}{1080} h y_{n}^{\prime}-\sum_{j=0}^{\infty} \frac{h^{j+1}}{j !} y_{n}^{j+1}\left\{\frac{32}{45}\left(\frac{1}{2}\right)^{j}+\frac{11}{120}(1)^{j}+\frac{8}{135}\left(\frac{3}{2}\right)^{j}-\frac{7}{360}(2)^{j}+\frac{1}{1080}(3)^{j}\right\} \\
\sum_{j=0}^{\infty} \frac{\left(\frac{3}{2}\right)^{j}}{j !} y_{n}^{j}-y_{n}-\frac{103}{640} h y_{n}^{\prime}-\sum_{j=0}^{\infty} \frac{h^{j+1}}{j !} y_{n}^{j+1}\left\{\frac{27}{40}\left(\frac{1}{2}\right)^{j}+\frac{243}{640}(1)^{j}+\frac{13}{40}\left(\frac{3}{2}\right)^{j}-\frac{27}{640}(2)^{j}+\frac{1}{640}(3)^{j}\right\} \\
\sum_{j=0}^{\infty} \frac{(2)^{j}}{j !} y_{n}^{j}-y_{n}-\frac{7}{45} h y_{n}^{\prime}-\sum_{j=0}^{\infty} \frac{h^{j+1}}{j !} y_{n}^{j+1}\left\{\frac{32}{45}\left(\frac{1}{2}\right)^{j}+\frac{4}{15}(1)^{j}+\frac{32}{45}\left(\frac{3}{2}\right)^{j}+\frac{7}{45}(2)^{j}+0(3)^{j}\right\} \\
\sum_{j=0}^{\infty} \frac{(3)^{j}}{j !} y_{n}^{j}-y_{n}-\frac{11}{40} h y_{n}^{\prime}-\sum_{j=0}^{\infty} \frac{h^{j+1}}{j !} y_{n}^{j+1}\left\{0\left(\frac{1}{2}\right)^{j}+\frac{81}{40}(1)^{j}-\frac{8}{5}\left(\frac{3}{2}\right)^{j}+\frac{81}{40}(2)^{j}+\frac{11}{40}(3)^{j}\right\}
\end{array}\right]=\left[\begin{array}{l}
0 \\
0 \\
0 \\
0 \\
0
\end{array}\right]
$$

Thus, $\bar{c}_{0}=\bar{c}_{1}=\bar{c}_{2}=\bar{c}_{3}=\bar{c}_{4}=\bar{c}_{5}=\bar{c}_{6}=\overline{0}$; implying that the order of the three-step method with two off-grid points is $p=\left[\begin{array}{ccccc}6 & 6 & 6 & 6 & 6\end{array}\right]^{T}$. That is, the method is of uniform order 6. It's error constant is given by

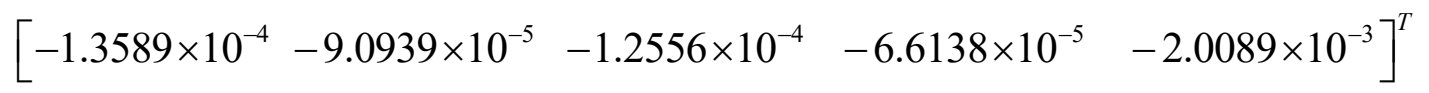




\section{B. Consistency of the Three-Step Hybrid Block Methods}

Recall that a method is consistent if it has order $p \geq 1$, [11]. Thus, the three-step hybrid block method with one off-grid point (11) is consistent since it is of uniform order $p=5 \geq 1$. Also, the three-step hybrid block method with two off-grid points (16) is consistent since it is of uniform order $p=6 \geq 1$. Consistency controls the magnitude of the local truncation error committed at each stage of the computation.

C. Zero-Stability of the Three-Step Hybrid Block Methods

Definition 2 [10]

$A$ block method is said to be zero-stable, if the roots $z_{s}, s=1,2, \ldots, k$ of the first characteristic polynomial $\rho(z)$ defined by $\rho(z)=\operatorname{det}\left(z A^{(0)}-E\right)$ satisfies $\left|z_{s}\right| \leq 1$ and every root satisfying $\left|z_{s}\right|=1$ have multiplicity not exceeding the order of the differential equation.

For the three-step method with one off-grid point (11), the first characteristic polynomial is given by,

$$
\rho(z)=\left|\left[\begin{array}{llll}
1 & 0 & 0 & 0 \\
0 & 1 & 0 & 0 \\
0 & 0 & 1 & 0 \\
0 & 0 & 0 & 1
\end{array}\right]-\left[\begin{array}{llll}
0 & 0 & 0 & 1 \\
0 & 0 & 0 & 1 \\
0 & 0 & 0 & 1 \\
0 & 0 & 0 & 1
\end{array}\right]\right|=\left|\begin{array}{llll}
z & 0 & 0 & -1 \\
0 & z & 0 & -1 \\
0 & 0 & z & -1 \\
0 & 0 & 0 & z-1
\end{array}\right|=z^{3}(z-1)
$$

Thus, solving for $z$ in

$$
z^{3}(z-1)=0
$$

gives $z_{1}=z_{2}=z_{3}=0$ and $z_{4}=1$. Hence, the three-step hybrid block method with one off-grid point is zero-stable. Applying the same procedure for the three-step hybrid block method with two off-grid points (16), we obtain $z_{1}=z_{2}=z_{3}=z_{4}=0$ and $z_{5}=1$, implying that it is also is zero-stable.

\section{Convergence of the Three-Step Hybrid Block Methods}

Theorem 2 [10]

The necessary and sufficient conditions for the linear multistep method to be convergent are that it be consistent and zero-stable.

Therefore, the two three-step methods derived in (11) and (16) are both convergent since they are consistent and zero-stable, [10].

\section{E. Regions of Absolute Stability of the Three-Step Hybrid Block Methods}

Applying the boundary locus method, the stability polynomial of the three-step hybrid block method with one off-grid point (11) is given by,

$$
\begin{aligned}
\bar{h}(w)= & -h^{4}\left(\frac{1}{8} w^{3}-\frac{1}{40} w^{4}\right)-h^{3}\left(\frac{23}{120} w^{4}+\frac{67}{120} w^{3}\right)+h^{2}\left(\frac{7}{10} w^{4}-\frac{13}{10} w^{3}\right) \\
& -h\left(\frac{13}{10} w^{4}+\frac{17}{10} w^{3}\right)+w^{4}-w^{3}
\end{aligned}
$$

Therefore, the region of absolute stability of the three-step hybrid block method with one off-grid point is shown in Fig. 1. 


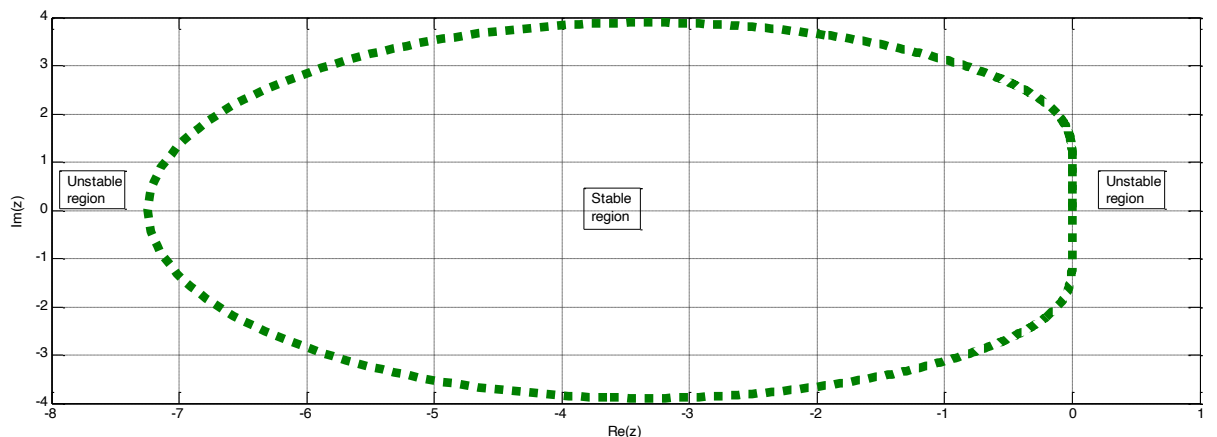

Fig. 1. Stability region of the three-step hybrid block method with one off-grid point.

The stability region obtained in Fig. 1 is A-stable. Note that the stability region is the interior of the curve.

On the other hand, the stability polynomial of the three-step hybrid block method with two off-grid points (16) is given by,

$$
\begin{aligned}
\bar{h}(w) & =-\left(\frac{1}{160} w^{5}+\frac{1}{32} w^{4}\right)+h^{4}\left(\frac{9}{160} w^{5}-\frac{29}{160} w^{4}\right)-h^{3}\left(\frac{13}{48} w^{5}+\frac{29}{48} w^{4}\right) \\
& +h^{2}\left(\frac{19}{24} w^{5}-\frac{31}{24} w^{4}\right)-h\left(\frac{4}{3} w^{5}+\frac{5}{3} w^{4}\right)+w^{5}-w^{4}
\end{aligned}
$$

Thus, the region of absolute stability of the three-step hybrid block method with two off-grid points is shown in Fig. 2.

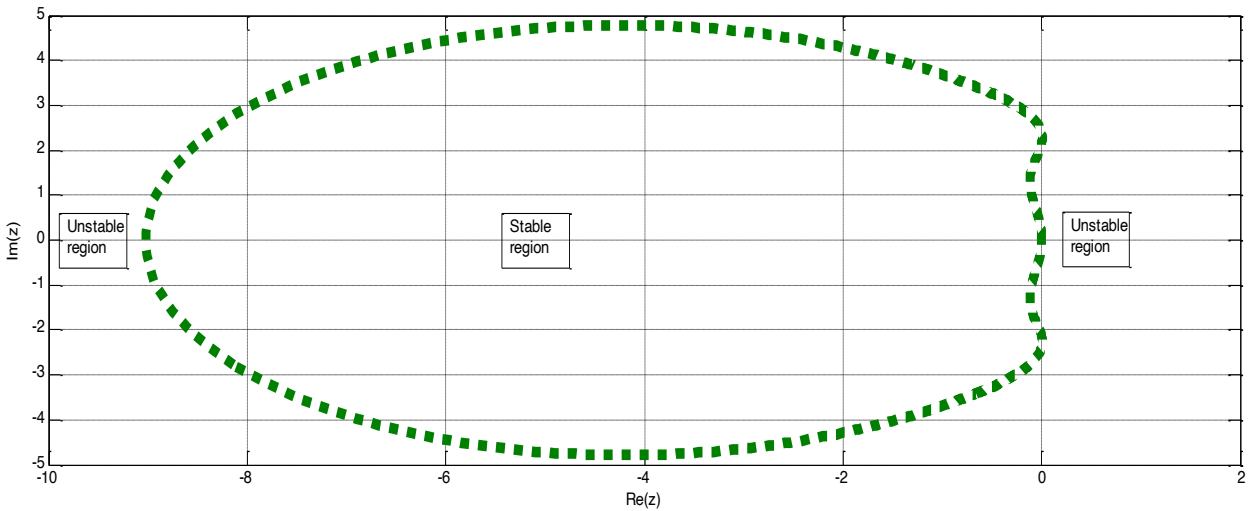

Fig. 2. Stability region of the three-step hybrid block method with two off-grid points.

The stability region obtained in Fig. 2 is also A-stable.

\section{NUMERICAL EXPERIMENTS AND DisCUSSION OF RESUlTS}

\section{A. Numerical Experiments}

The newly derived three-step hybrid block methods shall be applied in solving linear and nonlinear systems of equations of the form (1). From the numerical experiments, the results so obtained shall be presented in tabular form. The following notations shall be used in the tables below:

3SHM1O-Three-step hybrid block method with one off-grid point

3SHM2O-Three-step hybrid block method with two off-grid points

\section{Problem 1}

Consider the linear stiff system in the range $0 \leq x \leq 1$ solved by [4],

$$
\left[\begin{array}{l}
y_{1}{ }^{\prime}(x) \\
y_{2}{ }^{\prime}(x)
\end{array}\right]=\left[\begin{array}{rr}
-1 & 95 \\
-1 & -97
\end{array}\right]\left[\begin{array}{l}
y_{1}(x) \\
y_{2}(x)
\end{array}\right],\left[\begin{array}{l}
y_{1}(0) \\
y_{2}(0)
\end{array}\right]=\left[\begin{array}{l}
1 \\
1
\end{array}\right]
$$

whose exact solution is given by, 
$\left[\begin{array}{l}y_{1}(x) \\ y_{2}(x)\end{array}\right]=\frac{1}{47}\left[\begin{array}{l}95 e^{-2 x}-48 e^{-95 x} \\ 48 e^{-96 x}-e^{-2 x}\end{array}\right]$

The eigenvalues of the Jacobian matrix are $\lambda_{1}=-2, \lambda_{2}=-96$ with the stiffness ratio 1:48

TABLE I: COMPARISON OF THE ABSOLUTE ERRORS IN 3SHM1O AND 3SHM2O WITH THAT OF [4] FOR PROBLEM 1

\begin{tabular}{|c|c|c|c|c|}
\hline$h$ & $y_{\mathrm{i}}$ & $\begin{array}{c}\text { Error in } \\
3 \text { SHM1O }\end{array}$ & $\begin{array}{c}\text { Error in } \\
3 \mathrm{SHM} 2 \mathrm{O}\end{array}$ & Error in [4] \\
\hline \multirow[t]{2}{*}{0.0625} & $y_{1}$ & $\begin{array}{l}2.1998 \\
\times 10^{-15}\end{array}$ & $\begin{array}{l}4.1562 \\
\times 10^{-14}\end{array}$ & $3 \times 10^{-08}$ \\
\hline & $y_{2}$ & $\begin{array}{l}5.1273 \\
\times 10^{-13}\end{array}$ & $\begin{array}{l}8.7325 \\
\times 10^{-16}\end{array}$ & $4 \times 10^{-10}$ \\
\hline
\end{tabular}

Problem 1 was solved at the step size $h=0.0625$ in order to compare our result with that of [4]. The results obtained clearly depict that both 3SHM1O and 3SHM2O performed better than that of [4].

\section{Problem 2}

Consider the linear mildly stiff system,

$\left[\begin{array}{l}y_{1}{ }^{\prime}(x) \\ y_{2}{ }^{\prime}(x)\end{array}\right]=\left[\begin{array}{rr}998 & 1998 \\ -999 & -1999\end{array}\right]\left[\begin{array}{l}y_{1}(x) \\ y_{2}(x)\end{array}\right],\left[\begin{array}{l}y_{1}(0) \\ y_{2}(0)\end{array}\right]=\left[\begin{array}{l}1 \\ 1\end{array}\right]$

The exact solution of the linear system of equations above is given by,

$$
\left[\begin{array}{l}
y_{1}(x) \\
y_{2}(x)
\end{array}\right]=\left[\begin{array}{l}
4 e^{-x}-3 e^{-1000 x} \\
-2 e^{-x}+3 e^{-1000 x}
\end{array}\right]
$$

It is important to state that the eigenvalues of the Jacobian matrix are $\lambda_{1}=-1, \lambda_{2}=-1000$ with the stiffness ratio $1: 1000$.

TABLE II: COMPARISON OF THE ABSOLUTE ERRORS IN 3SHM1O AND 3SHM2O WITH THAT OF [2] FOR PROBLEM

\begin{tabular}{|c|c|c|c|c|}
\hline$x$ & $y_{\mathrm{i}}$ & $\begin{array}{c}\text { Error in } \\
3 \mathrm{SHM} 1 \mathrm{O}\end{array}$ & $\begin{array}{c}\text { Error in } \\
3 \mathrm{SHM} 2 \mathrm{O}\end{array}$ & Error in [2] \\
\hline \multirow{4}{*}{5} & \multirow[b]{2}{*}{$y_{1}$} & 1.2352 & 2.1372 & 1.3920 \\
\hline & & $\times 10^{-13}$ & $\times 10^{-15}$ & $\times 10^{-11}$ \\
\hline & \multirow[b]{2}{*}{$y_{2}$} & 2.3527 & 8.7325 & 6.9700 \\
\hline & & $\times 10^{-13}$ & $\times 10^{-16}$ & $\times 10^{-12}$ \\
\hline \multirow{4}{*}{40} & \multirow[b]{2}{*}{$y_{1}$} & 3.1562 & 2.3372 & 3.3628 \\
\hline & & $\times 10^{-16}$ & $\times 10^{-18}$ & $\times 10^{-12}$ \\
\hline & \multirow[b]{2}{*}{$y_{2}$} & 3.1625 & 4.1783 & 1.6818 \\
\hline & & $\times 10^{-16}$ & $\times 10^{-18}$ & $\times 10^{-12}$ \\
\hline \multirow{4}{*}{70} & \multirow[b]{2}{*}{$y_{1}$} & 4.2561 & 3.2891 & 3.9325 \\
\hline & & $\times 10^{-19}$ & $\times 10^{-21}$ & $\times 10^{-13}$ \\
\hline & \multirow[b]{2}{*}{$y_{2}$} & 3.6172 & 3.2891 & 1.4664 \\
\hline & & $\times 10^{-20}$ & $\times 10^{-22}$ & $\times 10^{-13}$ \\
\hline
\end{tabular}

\section{Problem 3}

Consider the well-known nonlinear two-dimensional Kaps problem in the range $0 \leq x \leq 20$ solved by [7],

$$
\left[\begin{array}{l}
y_{1}{ }^{\prime}(x) \\
y_{2}{ }^{\prime}(x)
\end{array}\right]=\left[\begin{array}{l}
-1002 y_{1}(x)+1000 y_{2}^{2}(x) \\
y_{1}(x)-y_{2}(x)\left(1+y_{2}(x)\right)
\end{array}\right],\left[\begin{array}{l}
y_{1}(0) \\
y_{2}(0)
\end{array}\right]=\left[\begin{array}{l}
1 \\
1
\end{array}\right]
$$


The exact solution is given by,

$\left[\begin{array}{l}y_{1}(x) \\ y_{2}(x)\end{array}\right]=\left[\begin{array}{l}e^{-2 x} \\ e^{-x}\end{array}\right]$

The absolute error at the $h$ the step size and $\mathrm{N}$ the number of computation steps are presented in Table III.

TABLE III: COMPARISON OF THE ABSOLUTE ERRORS IN 3SHM1O AND 3SHM2O WITH THAT OF [7] FOR PROBLEM

\begin{tabular}{|c|c|c|c|c|c|}
\hline$x$ & $N$ & $y_{\mathrm{i}}$ & $\begin{array}{c}\text { Error in } \\
3 \text { SHM1O }\end{array}$ & $\begin{array}{c}\text { Error in } \\
3 \mathrm{SHM} 2 \mathrm{O}\end{array}$ & Error in [7] \\
\hline \multirow[t]{2}{*}{5} & \multirow[t]{2}{*}{4} & $y_{1}$ & $\begin{array}{l}4.5627 \\
\times 10^{-11}\end{array}$ & $\begin{array}{l}1.6661 \\
\times 10^{-13}\end{array}$ & $\begin{array}{l}2.1670 \\
\times 10^{-09}\end{array}$ \\
\hline & & $y_{2}$ & $\begin{array}{l}3.3316 \\
\times 10^{-07}\end{array}$ & $\begin{array}{l}1.2112 \\
\times 10^{-10}\end{array}$ & $\begin{array}{l}1.3507 \\
\times 10^{-05}\end{array}$ \\
\hline \multirow[t]{2}{*}{1.25} & \multirow[t]{2}{*}{8} & $y_{1}$ & $\begin{array}{l}4.5471 \\
\times 10^{-11}\end{array}$ & $\begin{array}{l}1.6514 \\
\times 10^{-13}\end{array}$ & $\begin{array}{l}2.3329 \\
\times 10^{-09}\end{array}$ \\
\hline & & $y_{2}$ & $\begin{array}{l}3.3616 \\
\times 10^{-07}\end{array}$ & $\begin{array}{l}1.3507 \\
\times 10^{-10}\end{array}$ & $\begin{array}{l}2.8914 \\
\times 10^{-05}\end{array}$ \\
\hline \multirow[t]{2}{*}{0.833} & \multirow[t]{2}{*}{12} & $y_{1}$ & $\begin{array}{l}4.5317 \\
\times 10^{-11}\end{array}$ & $\begin{array}{l}1.6445 \\
\times 10^{-13}\end{array}$ & $\begin{array}{l}2.3078 \\
\times 10^{-09}\end{array}$ \\
\hline & & $y_{2}$ & $\begin{array}{l}3.5112 \\
\times 10^{-07}\end{array}$ & $\begin{array}{l}1.3722 \\
\times 10^{-10}\end{array}$ & $\begin{array}{l}2.9695 \\
\times 10^{-05}\end{array}$ \\
\hline \multirow[t]{2}{*}{0.625} & \multirow[t]{2}{*}{16} & $y_{1}$ & $\begin{array}{l}4.5164 \\
\times 10^{-11}\end{array}$ & $\begin{array}{l}1.6317 \\
\times 10^{-13}\end{array}$ & $\begin{array}{l}2.2987 \\
\times 10^{-09}\end{array}$ \\
\hline & & $y_{2}$ & $\begin{array}{l}3.4072 \\
\times 10^{-07}\end{array}$ & $\begin{array}{l}1.3978 \\
\times 10^{-10}\end{array}$ & $\begin{array}{l}2.9986 \\
\times 10^{-05}\end{array}$ \\
\hline \multirow[t]{2}{*}{0.5} & \multirow[t]{2}{*}{20} & $y_{1}$ & $\begin{array}{l}4.5090 \\
\times 10^{-11}\end{array}$ & $\begin{array}{l}1.6126 \\
\times 10^{-13}\end{array}$ & $\begin{array}{l}2.2948 \\
\times 10^{-09}\end{array}$ \\
\hline & & $y_{2}$ & $\begin{array}{l}3.4611 \\
\times 10^{-07}\end{array}$ & $\begin{array}{l}1.4155 \\
\times 10^{-10}\end{array}$ & $\begin{array}{l}3.0115 \\
\times 10^{-05}\end{array}$ \\
\hline
\end{tabular}

\section{Problem 4}

Consider the nonlinear system solved by [9],

$\left[\begin{array}{l}y_{1}{ }^{\prime}(x) \\ y_{2}{ }^{\prime}(x)\end{array}\right]=\left[\begin{array}{cc}-2 & 1 \\ 998 & -999\end{array}\right]\left[\begin{array}{l}y_{1}(x) \\ y_{2}(x)\end{array}\right]+\left[\begin{array}{l}2 \sin x \\ 999(\cos x-\sin x)\end{array}\right],\left[\begin{array}{l}y_{1}(0) \\ y_{2}(0)\end{array}\right]=\left[\begin{array}{l}2 \\ 3\end{array}\right]$

The exact solution is given by,

$\left[\begin{array}{l}y_{1}(x) \\ y_{2}(x)\end{array}\right]=\left[\begin{array}{l}2 e^{-x}+\sin x \\ 2 e^{-x}+\cos x\end{array}\right]$

The absolute error at the end point $x=10$ and $h$ the step size are presented in Table IV.

TABLE IV: COMPARISON OF THE ABSOLUTE ERRORS IN 3SHM1O AND 3SHM2O WITH THAT OF [9] FOR PROBLEM

\begin{tabular}{ccccc}
\hline \hline \multirow{2}{*}{$x$} & \multicolumn{5}{c}{4} \\
& $y_{\mathrm{i}}$ & Error in & Error in & Error in [9] \\
\hline \hline \multirow{2}{*}{0.25} & \multirow{2}{*}{$y_{1}$} & 1.15622 & 3.52617 & 4.50751 \\
& $\times 10^{-14}$ & $\times 10^{-15}$ & $\times 10^{-14}$ \\
& & 4.53625 & 3.57261 & 4.84057 \\
& $y_{2}$ & $\times 10^{-14}$ & $\times 10^{-15}$ & $\times 10^{-14}$ \\
& & & & \\
& & & & \\
0.5 & $y_{1}$ & & & \\
& & $\times 10^{-14}$ & $\times 10^{-15}$ & $\times 10^{-14}$
\end{tabular}




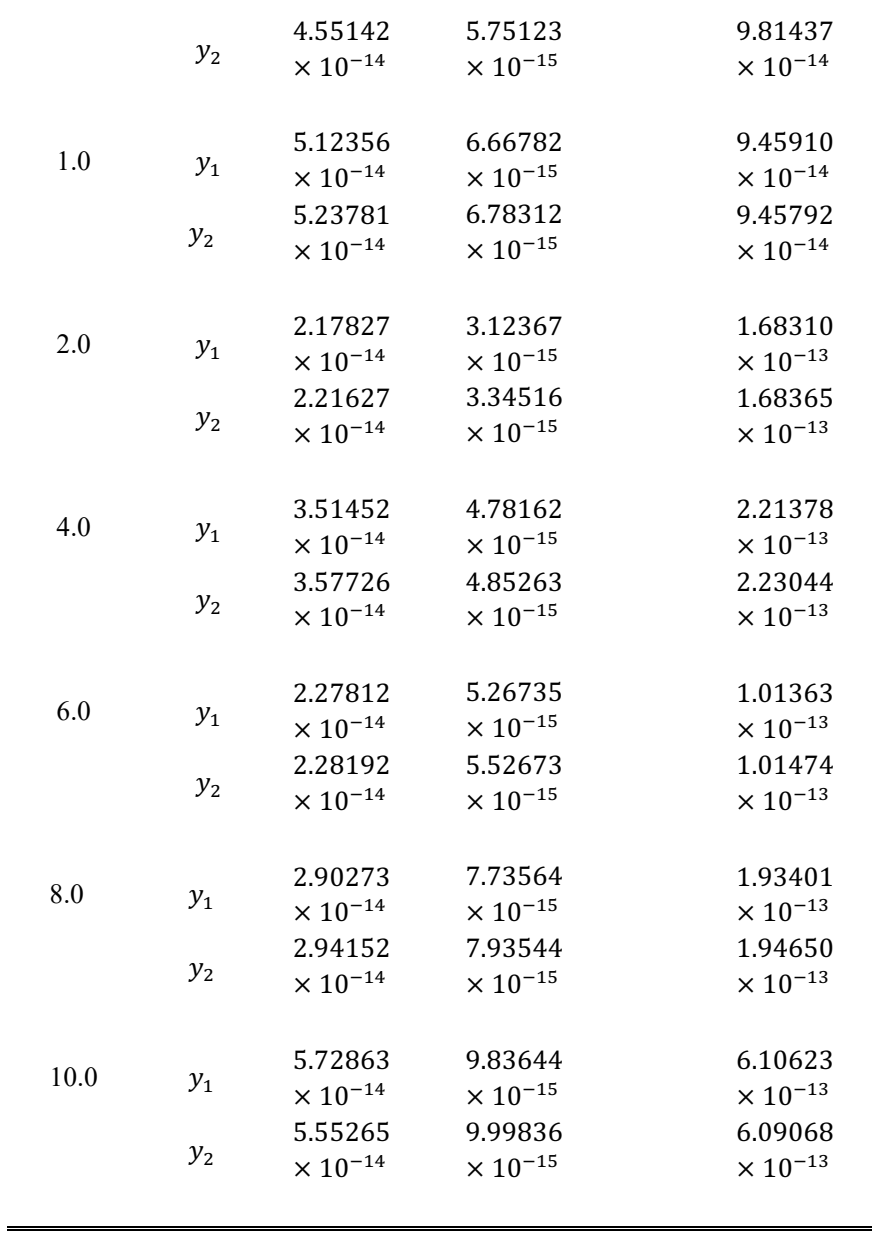

\section{B. Discussion of Results}

From the results presented in TABLES I-IV, it is clear that the two three-step hybrid block methods derived are efficient and computationally reliable in solving the linear and nonlinear stiff systems of the form (1). It is also clear that the three-step hybrid block method with two off-grid points (16) performed better than the three-step hybrid block method with one off-grid point (11). The stability regions obtained show that both methods are A-stable, see Fig. 1 and Fig. 2.

\section{CONCLUSION}

A pair of three-step hybrid block methods has been derived in this research. The methods derived were applied in solving linear and nonlinear systems of first order differential equations. The results obtained showed that the methods derived performed better than the ones with which we compared our results. The methods derived were also found to be stable, consistent, zero-stable and convergent.

\section{CONFLICT OF INTEREST}

Authors declare that they do not have any conflict of interest.

\section{REFERENCES}

[1] Henrici P. Discrete variable methods in ordinary differential equations. John Wiley \& Sons, New York; 1962.

[2] Rufai MA, Duromola MK, Ganiyu AA. Derivation of one-sixth hybrid block method for solving general first order ordinary differential equations. IOSR-Journal of Mathematics. 2016; 12: 20-27.

[3] Yakubu DG, Markus S. Second derivative of high order accuracy methods for the numerical integration of stiff initial value problems. Afrika Matematika. 2016; 27: 963-977.

[4] Adesanya AO, Onsachi RO, Odekunle MR. New algorithm for first order stiff initial value problems. Fasciculi Mathematici. 2017; 58: 19-28.

[5] Adesanya AO, Pantuvo TP, Umar D. On nonlinear methods for stiff and singular first order initial value problems. Nonlinear Analysis and Differential Equations. 2018; 6(2): 53-64.

[6] Khalsaraei MM, Shokri A, Molayi M. The new high approximation of stiff systems of first order initial value problems arising from chemical reactions by k-step L-stable hybrid methods. Iranian Journal of Mathematical Chemistry. 2019; 10(2): 181-193.

[7] Akinfenwa OA, Abdulganiy RI, Akinnukawe BI, Okunuga SA. Seventh order hybrid block method for solution of first order stiff systems of initial value problems. Journal of the Egyptian Mathematical Society. 2020; 28(34): 1-11.

[8] Ogunniran MO, Haruna Y, Adeniyi RB, Olayiwola MO. Optimized three-step hybrid block method for stiff problems in ordinary differential equations. Journal of Science and Engineering. 2020; 17(2): 80-95. 
[9] Akinnukawe BI, Muka KO. L-stable block hybrid numerical algorithm for first-order ordinary differential equations. Journal of the Nigerian Society of Physical Sciences. 2020; 2: 160-165.

[10] Lambert JD. Numerical methods for ordinary differential systems: The initial value problem, John Wiley and Sons LTD, United Kingdom; 1991.

[11] Fatunla SO. Numerical integrators for stiff and highly oscillatory differential equations. Mathematics of Computation. 1980; 34:373-390 